\title{
Overweight and obesity as potential prognostic factors for severity and mortality in patients with COVID-19: A living systematic review protocol
}

AUTHORS:

Catherine Bonilla-Untiveros (catherinbonilla@gmail.com)

ORCID: 0000-0002-1083-3386

Instituto Nacional de Salud (Perú), Red de Nutrición Basada en la Evidencia (Red NuBE)

Saby Camacho (sabycamacho@gmail.com)

ORCID: 0000-0003-3372-7719

Institución Nutrir México (México), Red de Nutrición Basada en la Evidencia (Red NuBE)

Eduard Baladia (e.baladia@academianutricion.org)

ORCID: 0000-0002-8123-8736

Red de Nutrición Basada en la Evidencia (Red NuBE), Academia Española de Nutrición y Dietética,

Pamplona, España.

Luis Ortiz-Muñoz (ortizkine@gmail.com)

ORCID: 0000-0001-6449-2153

UC Evidence Center, Cochrane Chile Associated Center, Pontificia Universidad Católica de Chile, Santiago, Chile

Gabriel Rada (radagabriel@epistemonikos.org)

ORCID: 0000-0003-2435-0710

Fundación Epistemonikos, Santiago, Chile

UC Evidence Center, Cochrane Chile Associated Center, Pontificia Universidad Católica de Chile, Santiago, Chile

Internal Medicine Department, Faculty of Medicine, Pontificia Universidad Católica de Chile, Santiago, Chile

COVID-19 L-OVE Working Group

Corresponding author:

Gabriel Rada

Email address:radagabriel@epistemonikos.org

Postal address: Holanda 895, Providencia, Santiago, Chile 


\section{ABSTRACT}

\section{Objective}

This living systematic review aims to provide a timely, rigorous and continuously updated summary about the impact of overweight or obesity as a prognostic factor for severity and mortality in patients with COVID-19.

\section{Design}

This is a protocol of a living systematic review.

\section{Data sources}

We will conduct searches in MEDLINE/PubMed, EMBASE, Cochrane Central Register of Controlled Trials (CENTRAL), grey literature and in a centralized repository in L.OVE (Living OVerview of Evidence). L.OVE is a platform that maps PICO questions to evidence from Epistemonikos database. In response to the COVID-19 emergency, L.OVE was adapted to expand the range of evidence it covers and customised to group all COVID-19 evidence in one place. The search will cover the period until the day before submission to a journal.

\section{Eligibility criteria for selecting studies and methods}

We adapted an already published common protocol for multiple parallel systematic reviews to the specificities of this question.

We will include all primary studies that assess patients with confirmed or suspected infection with SARS-CoV-2 and inform the relation of overweight or obesity with death or disease severity. Two reviewers will independently screen each study for eligibility, extract data, and assess the risk of bias. We will pool the results using meta-analysis and will apply the GRADE system to assess the certainty of the evidence for each outcome.

A living, web-based version of this review will be openly available during the COVID-19 pandemic. We will resubmit it every time the conclusions change or whenever there are substantial updates.

\section{Ethics and dissemination}

No ethics approval is considered necessary. The results of this review will be widely disseminated via peer-reviewed publications, social networks and traditional media.

\section{PROSPERO Registration}

Submitted to PROSPERO. Registration number: CRD42020198521

\section{Keywords}

COVID-19, severe acute respiratory syndrome coronavirus 2, Coronavirus Infections, Systematic review, obesity, overweight, body mass index, adiposity. 


\section{INTRODUCTION}

COVID-19 is an infection caused by the SARS-CoV-2 coronavirus(1). It was first identified in Wuhan, China, on December 31, 2019 (2). On March 11, 2020, WHO characterized the COVID-19 outbreak as a pandemic (1). Globally, as for July 2020, there have been 14,971,036 confirmed cases of COVID-19, including 618,017 deaths, reported to the World Health Organization (3).

While the majority of cases result in mild symptoms, some might progress to pneumonia, acute respiratory distress syndrome and death (4-6). The case fatality rate reported across countries, settings and age groups is highly variable, but it ranges from about $0.5 \%$ to $10 \%$ (7). In hospitalised patients it has been reported to be higher than $10 \%$ in some centres (8).

In relation to the COVID-19 outbreak, it is important to know about which patient groups are most at risk. Initial research identified people at greatest risk of developing severe forms of the disease and having a higher risk of death, such as older individuals, those with diabetes or cardiovascular, respiratory, or kidney disease. Currently, an increasing number of investigations have linked obesity to more severe COVID-19 illness and death (9).

Overweight and obesity are defined as abnormal or excessive fat accumulation that may impair health (10) . Body mass index (BMI) is used to define overweight and obesity. It is calculated as the ratio of weight in kilograms to the square of height in meters, expressed in units of $\mathrm{kg} / \mathrm{m}^{2}$ : a BMI $\geq 25$ and < 30 indicates overweight and $\geq 30$ indicates obesity. BMI is a useful population-level measure, because it strongly correlates with body fat percentage in adults.

The incidence of overweight and obesity has been on the rise for the last years, reaching over 2 billion people throughout the world (10). Nowadays, obesity itself could be considered a pandemic.

In a recent study, the risk for invasive mechanical ventilation in patients with COVID-19 infection admitted to intensive care was more than 7-fold higher for those BMI >35 compared to people with normal weight (BMI $<25 \mathrm{~kg} / \mathrm{m} 2)(11)$. Obesity is, also, associated with higher risk of ICU admission or death in patients with influenza A (H1N1) infection (12) .

The mechanisms by which obesity could be a risk factor for developing severe forms of COVID-19, are attributed to a decrease in lung function, modifications in the microbiota, increase in proinflammatory substances, and alterations in the immune response (13). Establishing overweight and obesity as prognostic factors for the disease could help to stratify patients according to their risk, and could support optimized management and resources utilization strategies to provide care to these patients.

Using innovative and agile processes, taking advantage of technological tools, and resorting to the collective effort of several research groups, this living systematic review aims to provide a timely, rigorous and continuously updated summary of the evidence available on the in patients with COVID19.

This living systematic review aims to provide a timely, rigorous and continuously updated summary of the evidence available about the impact of overweight or obesity in patients with COVID-19. 


\section{METHODS}

This manuscript complies with the 'Preferred Reporting Items for Systematic reviews and MetaAnalyses' (PRISMA-P) guidelines for reporting protocols of systematic reviews and meta-analysis (14) . Additionally we will follow the CHARM-PF checklist to determine which items to extract from primary studies of prognostic factors (15).

A protocol stating the shared objectives and methodology of multiple evidence syntheses (systematic reviews and overviews of systematic reviews) to be conducted in parallel for different questions relevant to COVID-19 was published elsewhere (16). This protocol was adapted to the specificities of the question assessed in this review and submitted to PROSPERO (Registration number: CRD42020198521)

\section{Search strategies}

\section{Electronic searches}

Our literature search was devised by the team maintaining the L.OVE platform (https://app.iloveevidence.com), using the following approach:

1. Identification of terms relevant to the population and exposure components of the search strategy, using Word2vec technology (17) to the corpus of documents available in Epistemonikos Database.

2. Discussion of terms with content and methods experts to identify relevant, irrelevant and missing terms.

3. Creation of a sensitive boolean strategy encompassing all the relevant terms

4. Iterative analysis of articles missed by the boolean strategy, and refinement of the strategy accordingly.

Our main search source will be Epistemonikos database (https://www.epistemonikos.org), a comprehensive database of systematic reviews and other types of evidence (18) that we supplemented with articles from multiple sources relevant to COVID-19 (19).

In sum, Epistemonikos Database acts as a central repository. Only articles fulfilling Epistemonikos criteria are visible by users. The remaining articles are only accessible for members of COVID-19 L.OVE Working Group.

We will conduct additional searches using highly sensitive searches in MEDLINE/PubMed, EMBASE, the Cochrane Central Register of Controlled Trials (CENTRAL), and grey literature.

The searches will cover from the inception date of each database until the day before submission. No study design, publication status or language restriction will be applied to the searches in Epistemonikos or the additional searches.

The following strategy will be used to search in Epistemonikos Database. We will adapt it to the syntax of other databases. 
(coronavir* OR coronovirus* OR "corona virus" OR "virus corona" OR "corono virus" OR "virus corono" OR hcov* OR "covid-19" OR covid19* OR "covid 19" OR "2019-nCoV" OR cv19* OR "cv-19" OR "cv 19" OR "n-cov" OR ncov* OR "sars-cov-2" OR "sars-cov2" OR (wuhan* AND (virus OR viruses OR viral)) OR (covid* AND (virus OR viruses OR viral)) OR "sars-cov" OR "sars cov" OR "sars-coronavirus" OR "severe acute respiratory syndrome" OR "mers-cov" OR "mers cov" OR "middle east respiratory syndrome" OR "middle-east respiratory syndrome" OR "covid-19-related" OR "SARS-CoV-2-related" OR "SARSCoV2-related" OR "2019-nCoV-related" OR "cv-19-related" OR "n-cov-related") AND (obes* OR obesity OR overweight OR BMI OR "unhealthy weight" OR fat OR "Body Mass Index" OR "body weight" OR kilograms OR kg OR pounds OR Ib* OR adiposity) AND (Risk factors OR risk* OR prognos* OR predictor* OR severity OR associat* OR comorbi*)

\section{Other sources}

In order to identify articles that might have been missed in the electronic searches, we will do the following:

1. Screen the reference lists of other systematic reviews and evaluate in full text all the articles they include.

2. Scan the reference lists of selected guidelines, narrative reviews and other documents.

3. Review the reference list of each included study.

\section{Eligibility criteria}

\section{Types of studies}

We will include primary studies that fulfill all the inclusion criteria. We will include prognostic factors and risk assessment model studies that are based on the typologies of prognosis proposed by lorio and colleagues (20), based on the PROGnosis RESearch Strategy (PROGRESS) Group framework (21). Studies evaluating excess weight (overweight or obesity) as a prognostic factor should use regression models adjusted for confounding variables.

We will exclude studies evaluating the effects on animal models or in vitro conditions. Studies based on statistical simulation or the use of artificial samples will also be excluded.

Types of participants

We will include studies assessing overweight or obesity as a prognostic factor in people with confirmed SARS-CoV-2 infection. Overweight or obesity will be clearly defined by the authors of the studies.

Whenever we find substantial clinical heterogeneity on how overweight or obesity was defined, we will explore it using a sensitivity analysis.

We will exclude studies if the population did not reflect the general population of interest, such as studies that included patients with other viral infections (e.g. SARS-CoV or MERS-CoV). 


\section{Type of outcomes}

We will not use the outcomes as an inclusion criteria during the selection process. Any article meeting all the criteria except for the outcome criterion will be preliminarily included and assessed in full text.

We will include studies that assess mortality or severe COVID-19 infection. We will accept the author's definitions of severe COVID-19 infection. Additionally, we will consider all the outcomes that can be assumed to reflect COVID-19 severity (i.e ICU requirement). We used the core outcome set COS-COVID (22), the existing guidelines and reviews and the judgement of the authors of this review as an input for selecting the primary and secondary outcomes, as well as to decide upon inclusion. The review team will revise this list of outcomes, in order to incorporate ongoing efforts to define Core Outcomes Sets (e.g. COVID-19 Core Outcomes (22) .

\section{Primary outcome}

- Risk of contracting COVID-19

- All-cause mortality

\section{Secondary outcomes}

- Mechanical ventilation support required and duration

- Extracorporeal membrane oxygenation

- Length of hospital stay

- Respiratory failure

- Score of clinical symptoms

- Time to SARS-CoV-2 RT-PCR negativity

- Risk for progression to severe illness

Other outcomes

- Acute respiratory distress syndrome

Primary and secondary outcomes will be presented in the GRADE 'Summary of Findings' tables (23), and a table with all the outcomes will be presented as an appendix.

\section{Selection of studies}

The results of the literature search in Epistemonikos database will be automatically incorporated into the L.OVE platform (automated retrieval), where they will be de-duplicated by an algorithm comparing unique identifiers (database ID, DOI, trial registry ID), and citation details (i.e. author names, journal, year of publication, volume, number, pages, article title and article abstract).

In L.OVE platform, two researchers will independently screen the titles and abstracts yielded by the search against the inclusion criteria. We will obtain the full reports for all titles that appear to meet the inclusion criteria or require further analysis to decide about their inclusion. 
We will record the reasons for excluding trials in any stage of the search and outline the study selection process in a PRISMA flow diagram adapted for the purpose of this project .

\section{Extraction and management of data}

Using standardised forms, two reviewers will independently extract data from each included study. We will collect the following information: study design, setting, participant characteristics (including disease severity, age, race, comorbidities, medication use) and study eligibility criteria; the outcomes assessed and the time they were measured; the source of funding of the study and the conflicts of interest disclosed by the investigators; the risk of bias assessment for each individual study.

We will resolve disagreements by discussion, and one arbiter will adjudicate unresolved disagreements.

\section{Risk of bias assessment}

We will use the Quality In Prognosis Studies tool to risk of bias assessment (24). Two authors will independently assess each included study in each domain, which includes items that authors will rate as low, medium or high risk of bias. We will provide an 'overall Risk of bias' for each domain by combining the items ratings.

We will not conduct an analysis of confounders because we are not assessing all prognostic factors in this review.

We will use A MeaSurement Tool to Assess systematic Reviews - 2 (AMSTAR-2) (25) to risk of bias assessment of systematic reviews.

If information is missing for making a risk of bias assessment, we will contact the authors of primary studies for further information. If they do not answer or if they are unable to give us needed information, we will rate the risk of bias as unclear. Whenever this happens, we will document our efforts to contact the study authors.

\section{Measures of association}

We will present the measure of association between weight status and the corresponding outcome. We will present the effect estimate as odds ratios (ORs) and their corresponding 95\% confidence intervals (Cl). In studies that report the measure of association as a hazard ratio (HR) or risk ratio (RR), we will convert them to ORs using the baseline risk (death rate or incidence of severe COVID-19 infection out of the total sample) reported in the studies $(4,26)$. We will use the crude effect estimates when the adjusted estimates are not provided. 


\section{Strategy for data synthesis}

We will present the results of the included studies including the individual frequencies in both tabular and narrative formats. When two or more studies show similar estimates for an outcome, we will meta-analyze all frequency measures. For outcomes where the above procedure is not possible, we will perform a qualitative analysis. If the measures among studies are uneven, we will analyse and explain those differences with a subgroup and sensitivity analysis if possible.

We will use inverse variance, random-effect models meta analysis to calculate an overall incidence rate for every individual outcome. For that purpose we will perform double arcsine transformation of individual incidence rates. All the calculations will be performed with the meta package for $\mathrm{R}$ software.

As we are not studying any intervention, we will not present summary effect measures.

\section{Subgroup and sensitivity analysis}

We will perform subgroup analysis according to the definition of overweight and obesity. In case we identify significant differences between subgroups (test for interaction $<0.05$ ) we will report the results of individual subgroups separately.

We will perform sensitivity analysis for different comorbidities presented by obesity people in included studies. We will also perform sensitivity analysis according to the disease severity, age, race, comorbidities, and medication use).

In cases where the primary analysis effect estimates and the sensitivity analysis effect estimates significantly differ we will either present the low risk of bias - adjusted sensitivity analysis estimates - or present the primary analysis estimates but downgrading the certainty of the evidence because of risk of bias.

\section{Assessment of certainty of evidence}

We will assess certainty of evidence for all the outcomes using a modified approach from the GRADE framework (20). We will consider risk of bias, inconsistency, indirectness, imprecision, publication bias, and publication bias. We will rank evidence as high, moderate, low or very low according to lorio et al. approach (20) and we will present the data in a 'GRADE Evidence.

\section{Living evidence synthesis}

An artificial intelligence algorithm deployed in the Coronavirus/COVID-19 topic of the L.OVE platform will provide instant notification of articles with a high likelihood to be eligible. The authors will review them, will decide upon inclusion, and will update the living web version of the review accordingly. We 
will consider resubmission to a journal if there is a change in the direction of the effect on the critical outcomes or a substantial modification to the certainty of the evidence. This review is part of a larger project set up to produce multiple parallel systematic reviews relevant to COVID-19 (16).

\section{NOTES}

\section{Acknowledgements}

The members of the COVID-19 L.OVE Working Group and Epistemonikos Foundation have made possible to build the systems and compile the information needed by this project. Epistemonikos is a collaborative effort, based on the ongoing volunteer work of over a thousand contributors since 2012.

\section{Roles and contributions}

GR conceived the common protocol for all the reviews being conducted by the COVID-19 L.OVE Working Group. CB and GR drafted the manuscript, and all other authors contributed to it. The corresponding author is the guarantor and declares that all authors meet authorship criteria and that no other authors meeting the criteria have been omitted.

The COVID-19 L.OVE Working Group was created by Epistemonikos and a number of expert teams in order to provide decision makers with the best evidence related to COVID-19. Up-to-date information about the group and its member organisations is available here: epistemonikos.cl/working-group

\section{Competing interests}

All authors declare no financial relationships with any organisation that might have a real or perceived interest in this work. There are no other relationships or activities that might have influenced the submitted work.

\section{Funding}

This project was not commissioned by any organisation and did not receive external funding.

Epistemonikos Foundation is providing training, support and tools at no cost for all the members of the COVID-19 L.OVE Working Group.

\section{PROSPERO registration}

This protocol has been submitted (Registration number: CRD42020198521)

\section{Ethics}

As researchers will not access information that could lead to the identification of an individual participant, obtaining ethical approval was waived. 


\section{Data sharing}

All data related to the project will be available. Epistemonikos Foundation will grant access to data. 
1. WHO. WHO Director-General's remarks at the media briefing on 2019-nCoV on 11 February 2020 [Internet]. 2020. Disponible en: https://www.who.int/dg/speeches/detail/who-director-generals-remarks-at-the-media-briefing-on-2019-ncov-on-11-february-2020

2. Hui DS, I Azhar E, Madani TA, Ntoumi F, Kock R, Dar O, et al. The continuing 2019-nCoV epidemic threat of novel coronaviruses to global health - The latest 2019 novel coronavirus outbreak in Wuhan, China. Int J Infect Dis IJID Off Publ Int Soc Infect Dis. 2020/01/14 ed. febrero de 2020;91:264-6.

3. World Health Organization. WHO Coronavirus Disease (COVID-19) Dashboard [Internet]. [citado 23 de julio de 2020]. Disponible en: https://covid19.who.int/

4. Wang Z. Converting odds ratio to relative risk in cohort studies with partial data information. J Stat Softw. 2013;55(5):1-11.

5. Tavakoli A, Vahdat K, Keshavarz M. Novel Coronavirus Disease 2019 (COVID-19): An Emerging Infectious Disease in the 21st Century. BPUMS. 1 de enero de 2020;22(6):432-50.

6. Li L-Q, Huang $T$, Wang $Y-Q$, Wang Z-P, Liang $Y$, Huang T-B, et al. COVID-19 patients' clinical characteristics, discharge rate, and fatality rate of meta-analysis. J Med Virol. junio de 2020;92(6):577-83.

7. CEBM Research. Global Covid-19 Case Fatality Rates [Internet]. 2020. Disponible en: https://www.cebm.net/covid-19/global-covid-19-case-fatality-rates/

8. Rodriguez-Morales AJ, Cardona-Ospina JA, Gutiérrez-Ocampo E, Villamizar-Peña R, Holguin-Rivera $Y$, Escalera-Antezana JP, et al. Clinical, laboratory and imaging features of COVID-19: A systematic review and meta-analysis. Travel Med Infect Dis. abril de 2020;34:101623.

9. Sattar N, McInnes IB, McMurray JJV. Obesity a Risk Factor for Severe COVID-19 Infection: Multiple Potential Mechanisms. Circulation. 22 de abril de 2020;

10. World Health Organization. Obesity and overweight [Internet]. 2020 [citado 31 de julio de 2020]. Disponible en: https://www.who.int/news-room/fact-sheets/detail/obesity-and-overweight

11. Simonnet A, Chetboun M, Poissy J, Raverdy V, Noulette J, Duhamel A, et al. High Prevalence of Obesity in Severe Acute Respiratory Syndrome Coronavirus-2 (SARS-CoV-2) Requiring Invasive Mechanical Ventilation. Obes Silver Spring Md. julio de 2020;28(7):1195-9.

12. Fezeu L, Julia C, Henegar A, Bitu J, Hu FB, Grobbee DE, et al. Obesity is associated with higher risk of intensive care unit admission and death in influenza $A$ (H1N1) patients: a systematic review and meta-analysis. Obes Rev. 1 de agosto de 2011;12(8):653-9.

13. Alberca RW, Oliveira L de M, Branco ACCC, Pereira NZ, Sato MN. Obesity as a risk factor for COVID19: an overview. Crit Rev Food Sci Nutr. 15 de junio de 2020;1-15.

14. Shamseer L, Moher D, Clarke M, Ghersi D, Liberati A, Petticrew M, et al. Preferred reporting items for systematic review and meta-analysis protocols (PRISMA-P) 2015: elaboration and explanation. BMJ. 2 de enero de 2015;349:g7647.

15. Moons KGM, de Groot JAH, Bouwmeester W, Vergouwe Y, Mallett S, Altman DG, et al. Critical appraisal and data extraction for systematic reviews of prediction modelling studies: the CHARMS checklist. PLoS Med. octubre de 2014;11(10):e1001744.

16. Rada G, Verdugo-Paiva F, Avila C, Morel-Matambio M, Bravo-Jeria R, Pesce F, et al. Evidence synthesis relevant to COVID-19: a protocol for multiple systematic reviews and overviews of 
systematic reviews. Medwave [Internet]. 1 de abril de 2020 [citado 19 de julio de 2020];20(3).

Disponible

en:

https://www.medwave.cl/link.cgi/Medwave/Estudios/Protocolos/7868?tab=ingles

17. Word2vec [Internet]. Github repository. [citado 19 de julio de 2020]. Disponible en: https://github.com/dperezrada/keywords2vec

18. Epistemonikos Database Methods [Internet]. Santiago: Epistemonikos Foundation. [citado 19 de julio de 2020]. Disponible en: https://www.epistemonikos.org/en/about_us/methods

19. Methods for the special L.OVE of Coronavirus infection [Internet]. Santiago: Epistemonikos Foundation. [citado 19 de julio de 2020]. Disponible en: https://app.iloveevidence.com/covid-19

20. Iorio A, Spencer FA, Falavigna M, Alba C, Lang E, Burnand B, et al. Use of GRADE for assessment of evidence about prognosis: rating confidence in estimates of event rates in broad categories of patients. BMJ. 16 de marzo de 2015;350:h870.

21. Hemingway H, Croft P, Perel P, Hayden JA, Abrams K, Timmis A, et al. Prognosis research strategy (PROGRESS) 1: a framework for researching clinical outcomes. BMJ. 5 de febrero de 2013;346:e5595.

22. COVID-19 Core Outcomes [Internet]. [citado 19 de julio de 2020]. Disponible en: https://www.covid-19-cos.org/

23. Guyatt GH, Oxman AD, Santesso N, Helfand M, Vist G, Kunz R, et al. GRADE guidelines: 12. Preparing summary of findings tables-binary outcomes. J Clin Epidemiol. febrero de 2013;66(2):158-72.

24. Hayden JA, van der Windt DA, Cartwright JL, Côté $P$, Bombardier C. Assessing bias in studies of prognostic factors. Ann Intern Med. 19 de febrero de 2013;158(4):280-6.

25. Shea BJ, Reeves BC, Wells G, Thuku M, Hamel C, Moran J, et al. AMSTAR 2: a critical appraisal tool for systematic reviews that include randomised or non-randomised studies of healthcare interventions, or both. BMJ. 21 de 2017;358:j4008.

26. Grant RL. Converting an odds ratio to a range of plausible relative risks for better communication of research findings. BMJ. 24 de enero de 2014;348. 